ARTICLE

DOI: $10.1038 / s 41467-017-01053-x$

\title{
Regulating $p$-block metals in perovskite nanodots for efficient electrocatalytic water oxidation
}

\author{
Bo-Quan Li ${ }^{1}$, Zi-Jing Xia ${ }^{1}$, Bingsen Zhang (i) ${ }^{2}$, Cheng Tang $^{1}$, Hao-Fan Wang ${ }^{1} \&$ Qiang Zhang (i) ${ }^{1}$
}

Water oxidation represents the core process of many sustainable energy systems, such as fuel cells, rechargeable metal-air batteries, and water splitting. Material surface defects with high-energy hanging bonds possess superb intrinsic reactivity, whose actual performance is limited by the dimension and conductivity of the electrocatalyst. Herein we propose a surface defect-rich perovskite electrocatalyst through a $p$-block metal regulation concept to achieve high performance for oxygen evolution. As a typical $p$-metal, $\mathrm{Sn}^{4+}$ dissolves from the solid phase from model SnNiFe perovskite nanodots, resulting in abundant surface defects with superior water oxidation performance. An oxygen pool model and a fusion-evolution mechanism are therefore proposed for the in-depth understanding of $p$-block metal regulation and the oxygen evolution reaction. The energy chemistry unveiled herein provides insights into water oxidation and helps to tackle critical issues in multi-electron oxygen electrocatalysis.

\footnotetext{
${ }^{1}$ Department of Chemical Engineering, Beijing Key Laboratory of Green Chemical Reaction Engineering and Technology, Tsinghua University, Beijing 100084 China. ${ }^{2}$ Shenyang National Laboratory for Material Science, Institute of Metal Research, Chinese Academy of Sciences, Shenyang 110016, China. Bo-Quan Li, Zi-Jing Xia, and Bingsen Zhang contributed equally to this work. Correspondence and requests for materials should be addressed to

Q.Z. (email: zhang-qiang@mails.tsinghua.edu.cn)
} 
E lectrocatalysis constitutes the core process of abundant sustainable energy systems such as fuel cells, rechargeable metal-air batteries, and water splitting ${ }^{1-3}$. However, classical electrochemical reactions (for instance, oxygen evolution reaction (OER), oxygen reduction reaction (ORR), hydrogen evolution reaction (HER), etc.) are very sluggish in kinetics and strongly inhibit the efficiency of the energy devices ${ }^{4-6}$. Decades of researches have been carried out in exploring high-performance electrocatalysts, including noble metal compounds ${ }^{7-9}$, transition metal hydroxides ${ }^{10-14}$, oxides ${ }^{15,16}$, perovskites ${ }^{17-21}$, sulfides $^{22-25}$, metal-free nanocarbon ${ }^{26-31}$, and their composites ${ }^{32-34}$. Insights into the rational design of efficient electrocatalysts are strongly desired in this field in order to avoid tedious trial and error exploration.

The intrinsic reactivity, the amount of exposed active sites, and the electrical conductivity are the most significant aspects for a well-established electrocatalyst with excellent reactivity ${ }^{35-37}$. It is widely accepted that surface defects possess high intrinsic reactivity toward electrocatalysis ${ }^{38-44}$. The electrochemical reactions only happen at the surface of the electrocatalysts where feedstock/ product can reach and electrons can be transferred during OER, ORR, and HER process. Surface defects with high-energy hanging bonds and dispersed empty electron orbitals are suitable for chemical adsorption and tend to interact strongly with reactants ${ }^{43}$.

Perovskites are a family of defect-abundant materials and exhibit excellent OER activity comparable to that of precious metal oxides ${ }^{19}, 45$. In a typical perovskite, A sites are usually alkaline earth metals, rare earth metals, or $p$-block metals while B sites are occupied with transition metals ${ }^{46-49}$. Both A sites and B sites are relatively easily substituted or dissociated ${ }^{50,51}$, which usually results in an oxygen non-stoichiometry and thus oxygen deficiency. Such oxygen deficiency provides abundant surface defects, which are believed to be the active sites for OER ${ }^{52-54}$. Meanwhile, the concept of nano-structured electrocatalysts affords high surface area and abundant active sites exposed to feedstocks, and is therefore highly expected to promote the overall electrochemical performance ${ }^{55-59}$. A stable conductive framework is also strongly considered to guarantee full demonstration of the intrinsic reactivity of electrocatalysts by reducing the resistance of electron transportation not only at the reactive interface, but also between the electrocatalysts and the current collectors $^{37,60}$

Based on this consideration, we introduced a unique strategy of $p$-block metal regulation of nano-sized perovskite electrocatalysts for OER. P-block metals possess a dual nature of metallicity and non-metallicity due to their unfilled $d$ orbitals. In a certain alkaline condition, $p$-block metal ions precipitate to form crystallized hydroxides appearing as a typical metal element. When the condition changes through the rise of alkalinity or addition of extra ligands, the $p$-block metal ions dissociate from the solid phase as soluble coordination complexes because of their non-metallicity, resulting in abundant vacant sites at the surface. Utilizing such unique properties of $p$-block metals, we can construct in situ defects with high intrinsic reactivity at the surface of perovskite electrocatalysts.

\section{Results}

Material synthesis and characterization. To prove the concept, $\mathrm{Sn}$ is selected as a typical $p$-block metal to regulate $\mathrm{SnNiFe}$ perovskite hydroxide nanodots in situ hybridized with mesoporous graphene framework $(\mathrm{MGF})^{61} \cdot \mathrm{Ni}^{2+}, \mathrm{Fe}^{3+}$, and $\mathrm{Sn}^{4+}$ co-precipitate into crystalized perovskite hydroxides spatially confined within the mesopores of graphene, exhibiting the structure of $\mathrm{SnNiFe}$ perovskite nanodots strongly coupled with graphene framework. As illustrated in Fig. 1, $\mathrm{Sn}^{4+}$ dissociates from the perovskite host and leaves in situ vacant sites at the surface during the $p$-block metal regulated electrochemical activation. The loss of $\mathrm{Sn}^{4+}$ affords abundant surface defects with high reactivity and facilitates the oxidation of transition metal ions into higher oxidative states at the same time, which are considered as critical active species toward OER ${ }^{62,63}$. The activated electrocatalyst exhibits efficient OER performance, superior to the state-of-art OER electrocatalyst $\mathrm{IrO}_{2}$ in aspects of reactivity, kinetics, and durability.

The conductive MGF support was fabricated by a templated chemical vapor deposition (CVD) method. Scanning electron microscopy (SEM) and transmission electron microscopy (TEM) images demonstrate a hierarchical structure of MGF with plentiful mesopores with a uniform diameter within $10 \mathrm{~nm}$ (Fig. 2a; Supplementary Fig. 1). The hybrid electrocatalyst of $\mathrm{SnNiFe}$ perovskite hydroxide nanodots with MGF (named as $\mathrm{n}-\mathrm{SnNiFe}$ ) was synthesized by in situ co-precipitation. No bulk perovskite is observed at macro-scale morphology characterization (Supplementary Fig. 2). High angle annular dark field scanning TEM (HAADF-STEM) image in Fig. 2b exhibits the uniform distribution of perovskite nanodots in 3D graphene scaffolds. Further morphology characterization indicates the $\mathrm{SnNiFe}$ perovskite is spatially confined within the mesopores of MGF (Fig. 2c), resulting in SnNiFe nanodots with an average diameter of ca. $5 \mathrm{~nm}$ (Fig. 2c, inserted). The as-synthesized perovskite nanodots are well crystalized, which were proved by high-resolution TEM (Fig. 2d) and X-ray diffraction (XRD) patterns (Fig. 2e). The lattice fringes observed in Fig. $2 \mathrm{~d}$ are identified to be (2 20 ), with the interplanar spacing being $0.269 \mathrm{~nm}$.

Energy-disperse X-ray spectroscopy (EDS), X-ray photoelectron spectroscopy (XPS), and inductively coupled plasma optical emission spectrometer (ICP) were carried out for element analysis. The atomic ratio of $\mathrm{Sn}:(\mathrm{NiFe})$ of $\mathrm{n}-\mathrm{SnNiFe}$ is close to 1 and Ni:Fe around 3.0 (Supplementary Fig. 3; Supplementary Table 1). The composition of $\mathrm{n}-\mathrm{SnNiFe}$ is consistent with the theoretical stoichiometric ratio identified by comprehensive characterization. EDS mapping exhibits the uniform distribution of every element (Supplementary Fig. 4). The successful fabrication of $\mathrm{n}$-SnNiFe perovskites attributes from the metallicity of transition metal and $p$-block metal. The $n$-SnNiFe with the structure of perovskite nanodots hybridized with conductive

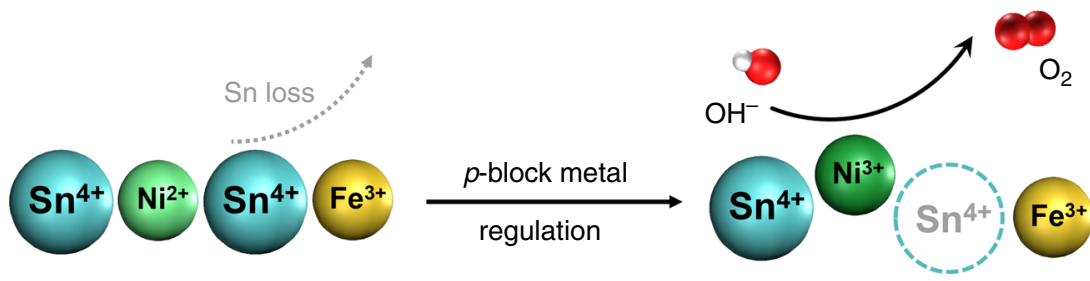

Fig. 1 Schematic of $p$-block metal regulation of perovskite electrocatalysts for OER. $\mathrm{Sn}^{4+}$ dissociates from SnNiFe perovskite host and leaves in situ vacant sites during the $p$-block metal regulation. The surface vacant sites possess high intrinsic reactivity for water oxidation 

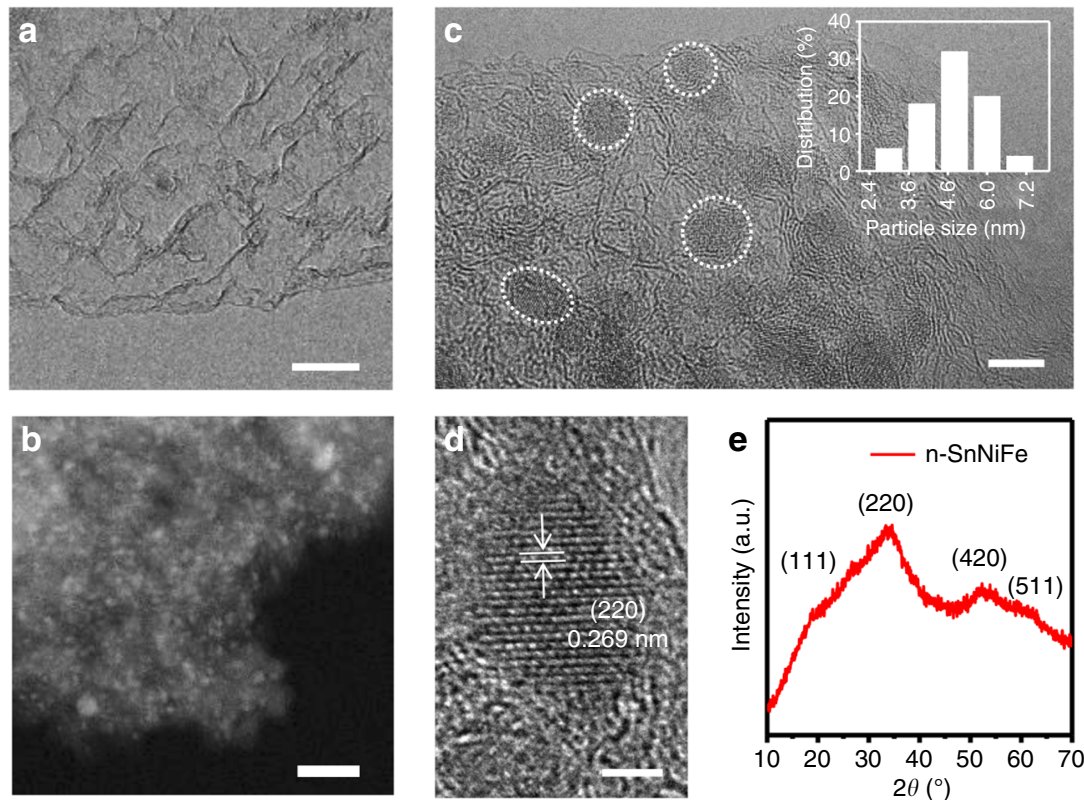

Fig. 2 Morphology and structure characterization of perovskite nanodots. a TEM image of MGF, exhibiting a mesoporous graphene framework. b HAADF-STEM image and c TEM image of $n$-SnNiFe peroskite electrocatalyst. Inset figure in $\mathbf{c}$ is the size distribution of $n$-SnNiFe perovskite nanodots. d The high-resolution TEM image and $\mathbf{e}$ XRD patterns of perovskite nanodots spatially confined in mesoporous graphene of $n$-SnNiFe electrocatalyst. Scale bars, $10 \mathrm{~nm}(\mathbf{a}), 5 \mathrm{~nm}(\mathbf{b}), 20 \mathrm{~nm}(\mathbf{c}), 2 \mathrm{~nm}$ (d)

MGF is expected to fully demonstrate the intrinsic OER reactivity of the electrocatalyst.

$P$-block metal activation and electrochemical evaluation. In order to create surface defects with intrinsic reactivity, $p$-block metal-regulated electrochemical activation of $n-S n N i F e$ perovskite nanodots was performed. $\mathrm{n}-\mathrm{SnNiFe}$ was electrochemically activated at a constant potential required to reach an initial current density of $5.0 \mathrm{~mA} \mathrm{~cm}^{-2}$ in $\mathrm{O}_{2}$-saturated $0.10 \mathrm{M}$ $\mathrm{KOH}$ for $300 \mathrm{~s}$. The $p$-block metal regulated $\mathrm{n}$-SnNiFe electrocatalyst after electrochemical activation is named as $\mathrm{p}-\mathrm{SnNiFe}$.

During the electrochemical activation, an obvious activation current was observed (Fig. 3a) with the current density raising from 4.3 to $4.5 \mathrm{~mA} \mathrm{~cm}^{-2}$. Figure $3 \mathrm{~b}$ demonstrates the $95 \%$ $i R$-compensated linear sweep voltammetry (LSV) profiles at a scan rate of $10.0 \mathrm{mV} \mathrm{s}^{-1}$. The current density of $\mathrm{p}-\mathrm{NiFeSn}$ perovskite electrocatalyst is distinctly increased compared with non-activated $\mathrm{n}-\mathrm{SnNiFe}$ perovskite nanodots. The overpotential of $\mathrm{p}-\mathrm{SnNiFe}$ at a current density of $10.0 \mathrm{~mA} \mathrm{~cm}^{-2}$ is $350 \mathrm{mV}$, which is among the best OER electrocatalysts (Supplementary Table 2) and even $20 \mathrm{mV}$ better than $\mathrm{IrO}_{2}$, which is recognized as the state-of-the-art OER electrocatalyst.

Tafel plots (Fig. 3c) exhibit the superiority of $\mathrm{p}-\mathrm{SnNiFe}$ perovskites $\left(35.0 \mathrm{mV} \mathrm{dec}^{-1}\right.$ ) over both $\mathrm{n}$-SnNiFe perovskites $\left(45.9 \mathrm{mV} \mathrm{dec}^{-1}\right)$ and $\mathrm{IrO}_{2}\left(134 \mathrm{mV} \mathrm{dec}^{-1}\right)$ with regard to OER kinetics. The stability of the electrocatalysts was characterized by a chronoamperometric method with an initial current density of $10.0 \mathrm{~mA} \mathrm{~cm}^{-2}$. The current density of $\mathrm{p}-\mathrm{SnNiFe}$ retains $60 \%$ after 20,000 s test while only $40 \%$ of the current density of $\mathrm{IrO}_{2}$ is preserved, indicating better stability of $\mathrm{p}$-SnNiFe perovskites against OER (Fig. 3d).

Bulk SnNiFe perovskite particles (named as b-SnNiFe) and the mechanical mixture of b-SnNiFe and MGF (named as b-SnNiFe + MGF) were prepared as control samples to demonstrate the significance of the unique pomegranate-like nanostructure of specially confined perovskites and highly conductive MGF toward both electrochemical activation and OER performance. The b-SnNiFe perovskite electrocatalyst exhibits a morphology of aggregated massive particles with an average diameter around $200 \mathrm{~nm}$ (Supplementary Fig. 5). The XRD patterns are identical with $\mathrm{n}$-SnNiFe perovskites (Supplementary Fig. 6), along with similar stoichiometric ratio of $\mathrm{Sn}, \mathrm{Ni}$, and $\mathrm{Fe}$ (Supplementary Fig. 7; Supplementary Table 1) and element distribution (Supplementary Fig. 8), indicating the smart in situ hybridized design with MGF contributes to the fabrication of nano structures without interfering the nature of $\mathrm{SnNiFe}$ perovskites. Bulk $\mathrm{SnNiFe}$ perovskite particles share identical chemical constitution and structure with $\mathrm{n}-\mathrm{SnNiFe}$, and are only different in morphology of perovskite and existence of MGF, making ready for illustration of the necessity of pomegranate-like nanostructures with nano-sized perovskites and highly conductive graphene toward effective electrocatalysis.

The electrochemical activation process of $b-\mathrm{SnNiFe}$ and b-SnNiFe + MGF was similar to that of $n-S n N i F e$ perovskites, and the $p$-block metal regulated samples after activation are named as pb-SnNiFe and $\mathrm{pb}-\mathrm{SnNiFe}+\mathrm{MGF}$, respectively. The OER reactivity of $b-\mathrm{SnNiFe}$ and $\mathrm{b}-\mathrm{SnNiFe}+\mathrm{MGF}$ electrocatalysts was enhanced, which was suggested by the raised current density during in situ electrochemical activation and decreased overpotential (Supplementary Figs. 9, 10). For instance, the overpotential at $10.0 \mathrm{~mA} \mathrm{~cm}^{-2}$ was $20 \mathrm{mV}$ decreased after electrochemical activation for $b-S n N i F e+M G F$. The improvement of OER reactivity indicates the universality of the $p$-block metal regulation strategy. However, the OER performance of neither pb-SnNiFe nor pb-SnNiFe + MGF is comparable with $\mathrm{p}-\mathrm{SnNiFe}$. The overpotential at $10.0 \mathrm{~mA} \mathrm{~cm}^{-2}$ of $\mathrm{p}$-SnNiFe is $110 \mathrm{mV}$ lower than that of pb-SnNiFe + MGF and far lower than pb-SnNiFe. In addition, the Tafel slope decreases from $190 \mathrm{mV} \mathrm{dec}^{-1}$ for pb-SnNiFe, $59.6 \mathrm{mV} \mathrm{dec}^{-1}$ for $\mathrm{pb}-\mathrm{SnNiFe}+$ MGF to $35.0 \mathrm{mV} \mathrm{dec}^{-1}$ for $\mathrm{p}-\mathrm{SnNiFe}$ (Supplementary Fig. 11). These evidences strongly confirm the superiority of $\mathrm{p}$-SnNiFe in OER reactivity and kinetics.

In order to evaluate the stability of $\mathrm{SnNiFe}$ perovskite hydroxides, long-time durability tests were performed on b-SnNiFe. Carbon nanotube (CNT, with a mass ratio of $20 \%$ ) was added to ensure the conductivity and the sample is named as 

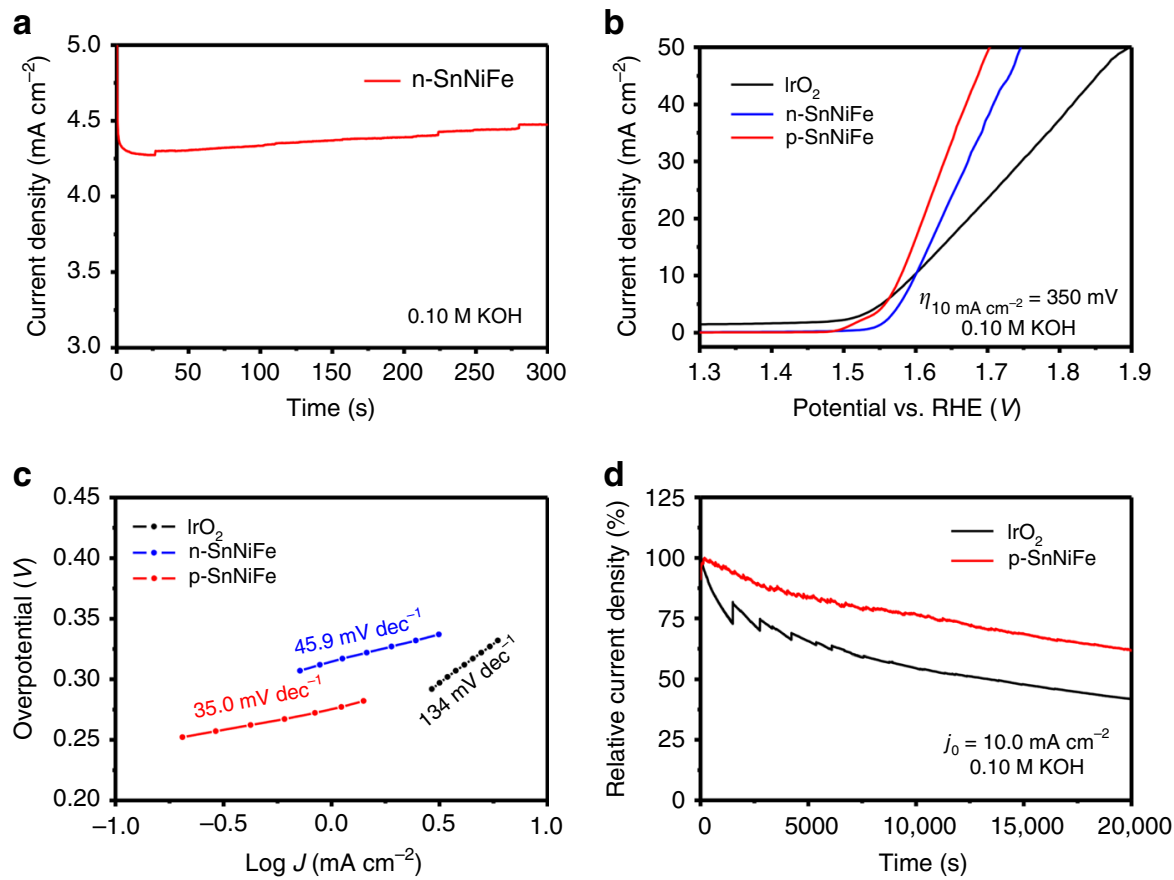

Fig. 3 Electrochemical activation and characterization of perovskite electrocatalysts for OER. a Electrochemical activation of $n$-SnNiFe perovskite at a constant voltage required to reach an initial current density of $5.0 \mathrm{~mA} \mathrm{~cm}^{-2}$ in $\mathrm{O}_{2}$-saturated $0.10 \mathrm{M} \mathrm{KOH}$. $\mathbf{b} 95 \%$ iR-compensated LSV profiles at a scan rate of $10.0 \mathrm{mV} \mathrm{s}^{-1}$ and $\mathbf{c}$ Tafel plots of $\mathrm{n}-\mathrm{SnNiFe}, \mathrm{p}-\mathrm{SnNiFe}$, and $\mathrm{IrO}_{2}$. $\mathbf{d}$ Chronoamperometric response at a constant potential required for an initial current density of $10.0 \mathrm{~mA} \mathrm{~cm}^{-2}$ of $\mathrm{p}-\mathrm{SnNiFe}$ perovskite and $\mathrm{IrO}_{2}$

a

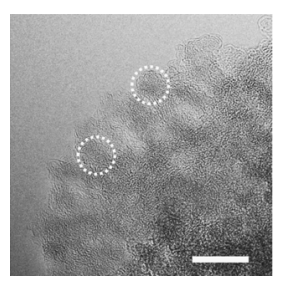

d

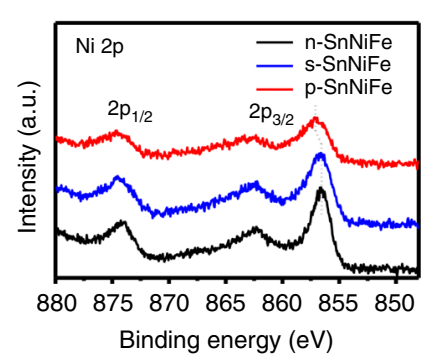

b

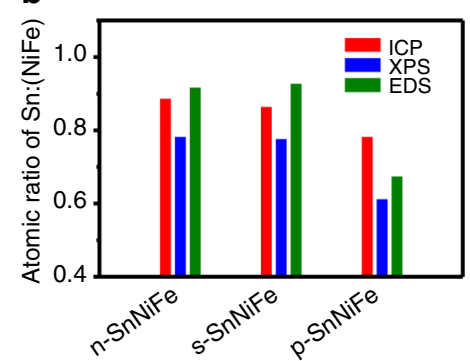

C

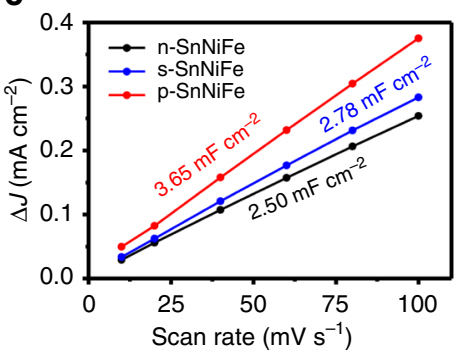

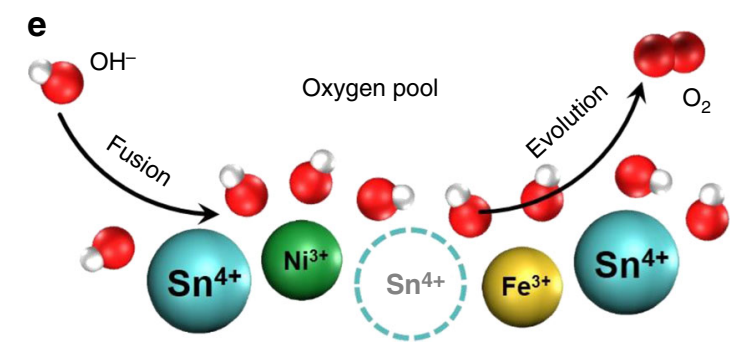

Fig. 4 Evaluation of the $p$-block regulation strategy. a High-resolution TEM image of p-SnNiFe, exhibiting no obvious difference from n-SnNiFe. $\mathbf{b}$ Atomic ratio of Sn:(NiFe) of $n-S n N i F e, s-S n N i F e$, and p-SnNiFe determined by ICP, XPS, and EDS. c ECSA and $\mathbf{d}$ high-resolution Ni 2p XPS spectra of $n-S n N i F e$, $\mathrm{s}-\mathrm{SnNiFe}$, and $\mathrm{p}-\mathrm{SnNiFe}$. e Scheme of the proposed oxygen pool model and fusion-evolution OER process. Scale bars, $10 \mathrm{~nm}$ (a)

b-SnNiFe + CNT. As is shown in Supplementary Fig. 12, the OER reactivity of b-SnNiFe $+\mathrm{CNT}$ electrocatalyst remains stable, with the overpotential at $10 \mathrm{~mA} \mathrm{~cm}^{-2}$ increases only $20 \mathrm{mV}$ after $10,000 \mathrm{~s}, 25 \mathrm{mV}$ after $20,000 \mathrm{~s}$, and $42 \mathrm{mV}$ after $40,000 \mathrm{~s}$ durability tests. The Tafel slopes exhibit similar tendency with slight increases. XRD patterns in Supplementary Fig. 13 and TEM images in Supplementary Fig. 14 further indicate the structure and morphology stability of perovskite hydroxides. The SnNiFe perovskite hydroxides exhibit satisfactory structural and electrochemical stability under OER condition in alkaline solution.

To further identify the origin of superiority of $\mathrm{p}$-SnNiFe over $\mathrm{pb}-\mathrm{SnNiFe}$ and $\mathrm{pb}-\mathrm{SnNiFe}+\mathrm{MGF}$, electrochemical impedance spectroscopy (EIS) and electrochemical active surface area (ECSA) analysis were carried out. The electrical conductivity of nano-sized SnNiFe perovskites in situ hybridized with MGF is better for both pristine and electrochemical activated 
electrocatalysts (Supplementary Fig. 15). In addition, MGF contributes to the promotion of ECSA exhibited in Supplementary Fig. 16, which is characterized by the double-layer capacity. Considering the ability of electrochemical activation and OER performance, the nano-sized perovskite and conductive framework play a critical role to enhance the OER performance for SnNiFe perovskites. The rational design of nanostructure and hybridization with conductive framework is of great significance for a well-established electrocatalyst.

Mechanism of $\boldsymbol{p}$-block metal regulation. In order to verify the surface defects attributed to $\mathrm{Sn}$ loss and understand the mechanism of $p$-block metal regulation of $n$-SnNiFe perovskites, detailed characterization of activated $\mathrm{p}$-SnNiFe perovskites was carried out. The s-SnNiFe perovskite was prepared by soaking $\mathrm{n}$-SnNiFe perovskite in the electrolyte as a control sample. There is no significant change between $\mathrm{p}-\mathrm{SnNiFe}$ and $\mathrm{n}-\mathrm{SnNiFe}$ in either morphology or crystal structure as shown in Fig. 4a, Supplementary Figs. 17, 18, suggesting morphology is not the key factor leading to OER activation. In addition, neither the electrical conductivity nor the surface hydrophility of the electrocatalysts is relevant confirmed by EIS in Supplementary Fig. 19 and Supplementary Table 3 and contact angel in Supplementary Fig. 20, respectively. However, element analysis exhibits consistent results of Sn loss by EDS, XPS, and ICP analysis (Supplementary Figs. 21, 22, and Supplementary Table 1). The atomic ratio of $\mathrm{Sn}$ :(NiFe) decreases from $\sim 0.9$ of $\mathrm{n}-\mathrm{SnNiFe}$ and $\mathrm{s}-\mathrm{SnNiFe}$ to $\sim 0.7$ of $\mathrm{p}-\mathrm{SnNiFe}$ (Fig. $4 \mathrm{~b}$ ), with the atomic ratio of $\mathrm{Ni}: \mathrm{Fe}$ remains relative stable around 3 (Supplementary Fig. 23). The dissociation of Sn lefts abundant vacant sites, which is confirmed by enhanced ECSA of $\mathrm{p}$-SnNiFe measured by capacitive current caused by adsorption and desorption of electrolyte (Fig. 4c).

Meanwhile, with the loss of positively charged $\mathrm{Sn}^{4+}$, transition metal ions in solid perovskite phase feel a stronger positive electric field. The transition metal ions tend to loss electron and therefore be oxidized to higher oxidative states. As shown in Fig. 4d, the high-resolution $\mathrm{Ni} 2 \mathrm{p}$ XPS spectra of $\mathrm{p}-\mathrm{SnNiFe}$ exhibit a $0.60 \mathrm{eV}$ shift to higher binding energy compared with $\mathrm{n}-\mathrm{SnNiFe}$ and $\mathrm{s}$-SnNiFe perovskites sharing similar Ni 2p XPS spectra. Such results strongly indicate the oxidation process and higher oxidative state of $\mathrm{Ni}$ in $\mathrm{p}$-SnNiFe perovskites, which is considered to contribute to the enhanced OER performance. In contrast, the oxidative states of $\mathrm{Fe}^{3+}$ and $\mathrm{Sn}^{4+}$ remain relative stable during the activation as illustrated in Supplementary Fig. 24 by XPS Fe 2 p spectra and Sn $3 p$ spectra. Above all, the unique dual properties of metallicity and non-metallicity of $\mathrm{Sn}$ is the main factor for construction of surface defects and oxidation of active transition metal ions, resulting in improved electrocatalysts and excellent OER performance.

\section{Discussion}

According to the discussion above, an oxygen pool model and a fusion-evolution mechanism are inferred and proposed for in-depth understanding of OER process. When $\mathrm{Sn}^{4+}$ dissolves during electrochemical activation, vacant sites are in situ created. Instead of staying at the original sites, lattice oxygen at the surface of the perovskite nanodots tends to form an amorphous layer with a more incompact oxygen arrangement, which is defined as the oxygen pool (Fig. 4e). Impelled by transition metal ions in high oxidative states, hydroxyl not only adsorbs on the surface of the electrocatalyst, but also fuses into the amorphous oxygen pool, identical to other hydroxyl in the amorphous solid state. The oxygen pool becomes crowded and excited with extra hydroxyl addition, which actuates electron transfer and product evolution, thus catalyzes the water oxidation. After the evolution of oxygen, the oxygen pool returns incompact and peaceful, and ready for another cycle of OER catalysis. Notably, such mechanism suggests the oxygen involved in product is not necessarily to be the same oxygen from the feedstock, but possibly comes from the lattice oxygen in a certain degree ${ }^{64,65}$. The oxygen pool model and the fusion-evolution process ingeniously explain the electrochemical activation and further inspire us for deeper understanding of OER and rational design of electrocatalysts.

In conclusion, we proposed the strategy of $p$-block metal regulation for rational design of electrocatalysts using the unique dual nature of metallicity and non-metallicity of $p$-block metal. The proof-of-concept SnNiFe perovskite nanodots spatially confined within MGF were fabricated. During the $p$-block metal regulated electrochemical activation, $\mathrm{Sn}^{4+}$ dissolves from solid phase, resulting in abundant surface defects with high intrinsic reactivity and transition metal ions oxidized to higher oxidative states favorable for OER catalysis. The activated perovskite electrocatalyst exhibits excellent OER performances, with an overpotential required for $10.0 \mathrm{~mA} \mathrm{~cm}^{-2}$ to be $350 \mathrm{mV}$ in alkaline electrolyte, which is comparable to the state-of-art $\mathrm{IrO}_{2}$ electrocatalyst. The creative oxygen pool model is proposed and describes that the amorphous oxygen surface after electrochemical activation is more inclined to reactant fusion and product evolution. Both the $p$-block metal regulation strategy and the oxygen pool model inspire us for rational electrocatalyst design and in-depth understanding of electrocatalysis. Therefore, the strategy of $p$-block metal regulation reported herein not only proves to be effective and rational for constructing defect-rich highly active electrocatalysts, but also enlightens in-depth understanding of mechanism of electrocatalysis.

\section{Methods}

Synthesis of MGF. MGF was prepared through a CVD method using mesoporous $\mathrm{MgO}$ as template and $\mathrm{CH}_{4} / \mathrm{NH}_{3}$ as carbon/nitrogen source, respectively. The $\mathrm{MgO}$ template was synthesized by a facile hydrothermal reaction using commercial MgO. Typically, 2.20 g polyethylene glycol (PEG)-2000 was dissolved in $100 \mathrm{~mL}$ deionized water and $1.0 \mathrm{~g} \mathrm{MgO}$ was added. The slurry was stirred for $48 \mathrm{~h}$ and then transferred into a $200 \mathrm{~mL}$ Teflon autoclave for hydrothermal reaction at $200{ }^{\circ} \mathrm{C}$ for $48 \mathrm{~h}$. The precipitate was filtered, washed with deionized water for three times and dried at $80^{\circ} \mathrm{C}$ overnight. The mesoporous $\mathrm{MgO}$ template was obtained after calcination of the as-obtained precursor at $650^{\circ} \mathrm{C}$ for $5.0 \mathrm{~h}$. The CVD growth was carried out in a furnace with $\mathrm{MgO}$ template placed in the middle of a horizontal quartz tube. The reactor was heated to $950{ }^{\circ} \mathrm{C}$ under Ar flow $\left(150 \mathrm{~mL} \mathrm{~min}^{-1}\right)$ with a heating rate of $20^{\circ} \mathrm{C} \mathrm{min}-1$. Both $\mathrm{CH}_{4}\left(70 \mathrm{~mL} \mathrm{~min}^{-1}\right)$ and $\mathrm{NH}_{3}\left(30 \mathrm{~mL} \mathrm{~min}^{-1}\right)$ were simultaneously introduced into the reactor for $15 \mathrm{~min}$ after the temperature was stable, and then the furnace was cooled naturally to room temperature under Ar protection. The product was purified using $6.0 \mathrm{~mol} \mathrm{~L}^{-1} \mathrm{HCl}$ at $95^{\circ} \mathrm{C}$ for $12 \mathrm{~h}$ to fully remove the $\mathrm{MgO}$ template. After filtering, washing with deionized water and ethanol, and freeze-drying for $24 \mathrm{~h}$, MGF was obtained for further synthesis.

Synthesis of perovskite electrocatalysts. $\mathrm{n}-\mathrm{SnNiFe}$ was synthesized by in situ co-precipitation. About $100 \mathrm{mg}$ MGF was dispersed into $30 \mathrm{~mL} \mathrm{~N}$-methylpyrrolidone (NMP) under sonication for $30 \mathrm{~min}$ to form a homogeneous suspension. About $1.5 \mathrm{mmol} \mathrm{Ni}\left(\mathrm{NO}_{3}\right)_{2} \cdot 6 \mathrm{H}_{2} \mathrm{O}, 0.50 \mathrm{mmol} \mathrm{Fe}\left(\mathrm{NO}_{3}\right)_{3} \cdot 9 \mathrm{H}_{2} \mathrm{O}$, and $2.0 \mathrm{mmol}$ citric acid were dissolved in $50 \mathrm{~mL}$ deionized water under stirring for $10 \mathrm{~min}$ and then mixed with the MGF slurry. About $10 \mathrm{~mL} \mathrm{SnCl}_{4}$ aqueous solution $\left(0.2 \mathrm{~mol} \mathrm{~L}^{-1}\right)$ was added into the above mixture under vigorous stirring. After that, $1.0 \mathrm{~mol} \mathrm{~L}^{-1}$ $\mathrm{NaOH}$ was added dropwise into the as-obtained solution under sonication until the $\mathrm{pH}$ reached 10 . The sonication went for another $30 \mathrm{~min}$ to accelerate the precipitation reaction. The product was separated by centrifuging at $10,000 \mathrm{rpm}$ for $10 \mathrm{~min}$ and purified by washing with deionized water and ethanol for three times, respectively. The $\mathrm{n}-\mathrm{SnNiFe}$ was finally obtained after freeze-drying for $24 \mathrm{~h}$ b-SnNiFe was synthesized under otherwise identical conditions without MGF. b-SnNiFe + MGF was prepared by simply mixing b-SnNiFe and MGF under grind.

Electrochemical activation of perovskite electrocatalysts. The electrochemical activation process was carried out using a three-electrode system. The samples for activation were prepared using the same method as the working electrode fabrication for electrochemical measurements. The activation conditions were identical with electrochemical evaluation process, with the electrolyte to be $\mathrm{O}_{2}$-saturated $0.10 \mathrm{M} \mathrm{KOH}$ (see more details in the electrocatalysis section). All 
samples were electrochemically activated at a constant potential required to reach a specific initial current density for $300 \mathrm{~s}$. n-SnNiFe and b-SnNiFe + MGF were activated at the initial current density of $5 \mathrm{~mA} \mathrm{~cm}^{-2}$, resulting in $\mathrm{p}-\mathrm{SnNiFe}$ and $\mathrm{pb}$ $\mathrm{SnNiFe}+\mathrm{MGF}$, respectively. The initial current density of b-SnNiFe was determined to be $2.5 \mathrm{~mA} \mathrm{~cm}^{-2}$ due to its poor reactivity and the final product was pb$\mathrm{SnNiFe}$ s-SnNiFe was prepared as control sample by simply soaking $\mathrm{n}$-SnNiFe into the electrolyte for $300 \mathrm{~s}$.

Characterization. The morphology was characterized using a JSM 7401F (JEOL Ltd., Tokyo, Japan) SEM at $3.0 \mathrm{kV}$ and a JEM 2010 (JEOL Ltd.) TEM at $120.0 \mathrm{kV}$. EDS analysis and mapping were performed using the JEM 2010 TEM equipped with an Oxford Instrument energy dispersive X-ray spectrometer. High-resolution transmission electron microscope (HRTEM) images were recorded using a FEI Tecnai $\mathrm{G}^{2}$ F20 TEM. XRD patterns were collected at $40.0 \mathrm{kV}$ and $120 \mathrm{~mA}$ with $\mathrm{Cu}-\mathrm{K}_{\alpha}$ radiation on Bruker D8 Advanced Diffractometer. XPS measurements were carried out by Escalab 250xi, with all XPS spectra corrected using C 1s line at $284.6 \mathrm{eV}$. Elemental analysis was performed using an inductively coupled plasma optical emission spectrometer (IRIS Intrepid II XSP, ThermoFisher, USA). The contact angle was measured using an OCAH200 Optical contact angle measuring instrument (Dataphysics, Germany).

Electrocatalysis. Electrochemical measurements were performed using a three-electrode system controlled by a CHI 760D electrochemistry station $(\mathrm{CH}$ Instrument, USA). A platinum sheet electrode served as the counter electrode. A saturated calomel electrode (SCE) was used as the reference electrode. The working electrode was a rotating disk electrode with a disk diameter of $5.0 \mathrm{~mm}$.

The working electrode was fabricated using the following method: $5.0 \mathrm{mg}$ electrocatalyst was dispersed in $0.95 \mathrm{~mL}$ ethanol and $0.05 \mathrm{~mL}$ Nafion solution $(5.0 \mathrm{wt} \%)$ and then sonicated for $30 \mathrm{~min}$ to form a homogeneous suspension. In total, $10.0 \mu \mathrm{L}$ suspension was dropped onto the glass carbon disk electrode, which was polished in advance. The working electrode was ready for electrochemical measurements or activation after the solvent was evaporated. The areal loading mass of the working electrode was $0.25 \mathrm{mg} \mathrm{cm}^{-2}$. The working electrode of electrochemical activated perovskites was directly used for further electrochemical measurements.

All electrochemical measurements were carried out in $\mathrm{O}_{2}$-saturated $0.10 \mathrm{~mol} \mathrm{~L}^{-1}$ $\mathrm{KOH}$ electrolyte at room temperature. The working electrode was rotated at $1600 \mathrm{rpm}$ during the tests. All potentials measured were corrected to reversible hydrogen electrode (RHE) using the following equation: $E_{\mathrm{RHE}}=E_{\mathrm{SCE}}+0.241 \mathrm{~V}+$ $0.0592 \mathrm{pH}$.

OER performance of the electrocatalysts was evaluated by LSV at a scan rate of $10.0 \mathrm{mV} \mathrm{s}^{-1}$. All polarization profiles were corrected with $95 \% i R$ compensation. Tafel slopes were calculated based on LSV curves using the Tafel equation $\eta=b \log \left(j / j_{0}\right) . \eta$ is the overpotential calculated using the following equation: $\eta=E_{\mathrm{RHE}}-1.23 \mathrm{~V}, b$ is the Tafel slope, $j$ is the current density, and $j_{0}$ is the exchange current density. ECSA was evaluated by the double-layer capacitance $\left(C_{\mathrm{dl}}\right)$, which was determined by capacitive current dependent on the scan rate of cyclic voltammetry $(\mathrm{CV})$. The $\mathrm{CV}$ measurements were carried out at a scan window from 0.000 to $0.050 \mathrm{~V}$ vs. SCE with scan rates of $10,20,40,60,80$, and $100 \mathrm{mV} \mathrm{s}^{-1}$. The double-layer capacitance was half of the slope calculated by fitting the $\Delta j=j_{a}-j_{b}$ at $0.025 \mathrm{~V}$ vs. SCE against the scan rate, which is used to represent the ECSA. The EIS was carried out at $0.57 \mathrm{~V}$ vs. SCE over a frequency range from 0.10 to $100 \mathrm{kHz}$ at the sinusoidal voltage amplitude of $5.0 \mathrm{mV}$. The stability test of the electrocatalysts was characterized at a constant voltage required to reach an initial current density of $10.0 \mathrm{~mA} \mathrm{~cm}{ }^{-2}$.

Data availability. All data generated or analysed during this study are included in this published article (and its Supplementary Information files).

Received: 7 February 2017 Accepted: 14 August 2017

Published online: 16 October 2017

\section{References}

1. Grande, L. et al. The lithium/air battery: still an emerging system or a practical reality? Adv. Mater. 27, 784-800 (2015).

2. Chu, S., Cui, Y. \& Liu, N. The path towards sustainable energy. Nat. Mater. 16, 16-22 (2017).

3. Hong, W. T. et al. Toward the rational design of non-precious transition metal oxides for oxygen electrocatalysis. Energy Environ. Sci. 8, 1404-1427 (2015).

4. Jiao, Y., Zheng, Y., Jaroniec, M. \& Qiao, S. Z. Design of electrocatalysts for oxygen- and hydrogen-involving energy conversion reactions. Chem. Soc. Rev. 44, 2060-2086 (2015).

5. McCrory, C. C. L., Jung, S., Peters, J. C. \& Jaramillo, T. F. Benchmarking heterogeneous electrocatalysts for the oxygen evolution reaction. J. Am. Chem. Soc. 135, 16977-16987 (2013).
6. Debe, M. K. Electrocatalyst approaches and challenges for automotive fuel cells. Nature 486, 43-51 (2012).

7. Lee, Y., Suntivich, J., May, K. J., Perry, E. E. \& Shao-Horn, Y. Synthesis and activities of rutile $\mathrm{IrO}_{2}$ and $\mathrm{RuO}_{2}$ nanoparticles for oxygen evolution in acid and alkaline solutions. J. Phys. Chem. Lett. 3, 399-404 (2012).

8. Zhang, N. et al. Superior bifunctional liquid fuel oxidation and oxygen reduction electrocatalysis enabled by ptnipd core-shell nanowires. Adv. Mater 29, 1603774 (2017)

9. Diaz-Morales, O. et al. Iridium-based double perovskites for efficient water oxidation in acid media. Nat. Commun. 7, 12363 (2016).

10. Zhu, X. et al. Monolithic-structured ternary hydroxides as freestanding bifunctional electrocatalysts for overall water splitting. J. Mater. Chem. A 4 , 7245-7250 (2016).

11. Gong, M. et al. An advanced Ni-Fe layered double hydroxide electrocatalyst for water oxidation. J. Am. Chem. Soc. 135, 8452-8455 (2013).

12. Lu, X. \& Zhao, C. Electrodeposition of hierarchically structured threedimensional nickel-iron electrodes for efficient oxygen evolution at high current densities. Nat. Commun. 6, 6616 (2015).

13. Zhang, B. et al. Homogeneously dispersed multimetal oxygen-evolving catalysts. Science 352, 333-337 (2016).

14. Tang, C., Wang, H.-F., Zhu, X.-L., Li, B.-Q. \& Zhang, Q. Advances in hybrid electrocatalysts for oxygen evolution reactions: rational integration of $\mathrm{NiFe}$ layered double hydroxides and nanocarbon. Part. Part. Syst. Char. 33, 473-486 (2016).

15. Han, L., Dong, S. \& Wang, E. Transition-metal (Co, Ni, and Fe)-based electrocatalysts for the water oxidation reaction. Adv. Mater. 28, 9266-9291 (2016).

16. Qi, J. et al. Porous nickel-iron oxide as a highly efficient electrocatalyst for oxygen evolution reaction. Adv. Sci. 2, 1500199 (2015).

17. Yagi, S. et al. Covalency-reinforced oxygen evolution reaction catalyst. Nat. Commun. 6, 8249 (2015).

18. Grimaud, A. et al. Double perovskites as a family of highly active catalysts for oxygen evolution in alkaline solution. Nat. Commun. 4, 2439 (2013).

19. Suntivich, J., May, K. J., Gasteiger, H. A., Goodenough, J. B. \& Shao-Horn, Y. A perovskite oxide optimized for oxygen evolution catalysis from molecular orbital principles. Science 334, 1383-1385 (2011).

20. Fabbri, E., Nachtegaal, M., Cheng, X. \& Schmidt, T. J. Superior bifunctional electrocatalytic activity of $\mathrm{Ba}_{0.5} \mathrm{Sr}_{0.5} \mathrm{Co}_{0.8} \mathrm{Fe}_{0.2} \mathrm{O}_{3-\delta} /$ carbon composite electrodes: insight into the local electronic structure. Adv. Energy Mater. 5, 1402033 (2015).

21. Zhou, W., Zhao, M. W., Liang, F. L., Smith, S. C. \& Zhu, Z. H. High activity and durability of novel perovskite electrocatalysts for water oxidation. Mater. Horiz. 2, 495-501 (2015).

22. You, B. \& Sun, Y. Hierarchically porous nickel sulfide multifunctional superstructures. Adv. Energy Mater. 6, 1502333 (2016).

23. Zhang, J. et al. Interface engineering of $\mathrm{MoS}_{2} / \mathrm{Ni}_{3} \mathrm{~S}_{2}$ heterostructures for highly enhanced electrochemical overall-water-splitting activity. Angew. Chem. Int. Ed. 55, 6701-6706 (2016).

24. Zhou, W. et al. $\mathrm{Ni}_{3} \mathrm{~S}_{2}$ nanorods/Ni foam composite electrode with low overpotential for electrocatalytic oxygen evolution. Energy Environ. Sci. 6, 2921-2924 (2013).

25. Long, X. et al. Metallic iron-nickel sulfide ultrathin nanosheets as a highly active electrocatalyst for hydrogen evolution reaction in acidic media. J. Am. Chem. Soc. 137, 11900-11903 (2015).

26. Wang, D.-W. \& Su, D. Heterogeneous nanocarbon materials for oxygen reduction reaction. Energy Environ. Sci. 7, 576-591 (2014).

27. Liang, Y., Li, Y., Wang, H. \& Dai, H. Strongly coupled inorganic/nanocarbon hybrid materials for advanced electrocatalysis. J. Am. Chem. Soc. 135 , 2013-2036 (2013).

28. Yang, H. B. et al. Identification of catalytic sites for oxygen reduction and oxygen evolution in $\mathrm{N}$-doped graphene materials: development of highly efficient metal-free bifunctional electrocatalyst. Sci. Adv. 2, e1501122 (2016).

29. Chen, S., Duan, J. J., Jaroniec, M. \& Qiao, S. Z. Nitrogen and oxygen dual-doped carbon hydrogel film as a substrate-free electrode for highly efficient oxygen evolution reaction. Adv. Mater. 26, 2925-2930 (2014).

30. Zheng, Y., Jiao, Y. \& Qiao, S. Z. Engineering of carbon-based electrocatalysts for emerging energy conversion: from fundamentality to functionality. Adv. Mater. 27, 5372-5378 (2015).

31. Tian, G. L. et al. Toward full exposure of "active sites": nanocarbon electrocatalyst with surface enriched nitrogen for superior oxygen reduction and evolution reactivity. Adv. Funct. Mater. 24, 5956-5961 (2014).

32. Ping, J. et al. Self-assembly of single-layer coal-layered double hydroxide nanosheets on 3D graphene network used as highly efficient electrocatalyst for oxygen evolution reaction. Adv. Mater. 28, 7640-7645 (2016).

33. Long, X. et al. A strongly coupled graphene and FeNi double hydroxide hybrid as an excellent electrocatalyst for the oxygen evolution reaction. Angew. Chem. Int. Ed. 53, 7584-7588 (2014). 
34. Wang, Y. C. et al. Bio-inspired leaf-mimicking nanosheet/nanotube heterostructure as a highly efficient oxygen evolution catalyst. Adv. Sci. 2, 1500003 (2015).

35. Zhang, G., Xia, B. Y., Wang, X. \& Lou, X. W. Strongly coupled $\mathrm{NiCo}_{2} \mathrm{O}_{4}-\mathrm{rGO}$ hybrid nanosheets as a methanol-tolerant electrocatalyst for the oxygen reduction reaction. Adv. Mater. 26, 2408-2412 (2014).

36. Stamenkovic, V. R., Strmcnik, D., Lopes, P. P. \& Markovic, N. M. Energy and fuels from electrochemical interfaces. Nat. Mater. 16, 57-69 (2017).

37. Tang, C. et al. Spatially confined hybridization of nanometer-sized NiFe hydroxides into nitrogen-doped graphene frameworks leading to superior oxygen evolution reactivity. Adv. Mater. 27, 4516-4522 (2015).

38. Dou, S., Tao, L., Huo, J., Wang, S. \& Dai, L. Etched and doped $\mathrm{Co}_{9} \mathrm{~S}_{8} /$ graphene hybrid for oxygen electrocatalysis. Energy Environ. Sci. 9, 1320-1326 (2016).

39. Ye, G. et al. Defects engineered monolayer $\mathrm{MoS}_{2}$ for improved hydrogen evolution reaction. Nano Lett. 16, 1097-1103 (2016).

40. Seitz, L. C. et al. A highly active and stable $\mathrm{IrO}_{\mathrm{x}} / \mathrm{SrIrO}_{3}$ catalyst for the oxygen evolution reaction. Science 353, 1011-1014 (2016).

41. Tang, C. et al. Topological defects in metal-free nanocarbon for oxygen electrocatalysis. Adv. Mater. 28, 6845-6851 (2016).

42. Tang, C. \& Zhang, Q. Nanocarbon for oxygen reduction electrocatalysis: dopants, edges, and defects. Adv. Mater. 29, 1604103 (2017).

43. Tao, L. et al. Edge-rich and dopant-free graphene as a highly efficient metal-free electrocatalyst for the oxygen reduction reaction. Chem. Commun. $\mathbf{5 2}$, 2764-2767 (2016)

44. Jiang, Y. F. et al. Significant contribution of intrinsic carbon defects to oxygen reduction activity. ACS Catal. 5, 6707-6712 (2015).

45. Gupta, S. et al. Bifunctional perovskite oxide catalysts for oxygen reduction and evolution in alkaline media. Chem. Asian J. 11, 10-21 (2016).

46. $\mathrm{Zhu}$, Y. et al. $\mathrm{SrNb}_{0.1} \mathrm{Co}_{0.7} \mathrm{Fe}_{0.2} \mathrm{O}_{3-\delta}$ perovskite as a next-generation electrocatalyst for oxygen evolution in alkaline solution. Angew. Chem. Int. Ed. 54, 3897-3901 (2015).

47. $\mathrm{Xu}, \mathrm{X}$. et al. Toward enhanced oxygen evolution on perovskite oxides synthesized from different approaches: a case study of $\mathrm{Ba}_{0.5} \mathrm{Sr}_{0.5} \mathrm{Co}_{0.8} \mathrm{Fe}_{0.2} \mathrm{O}_{3-\delta}$. Electrochim. Acta 219, 553-559 (2016).

48. Lee, J. G. et al. A new family of perovskite catalysts for oxygen-evolution reaction in alkaline media: $\mathrm{BaNiO}_{3}$ and $\mathrm{BaNi}_{0.83} \mathrm{O}_{2.5}$. J. Am. Chem. Soc. 138, 3541-3547 (2016).

49. Mefford, J. T. et al. Water electrolysis on $\mathrm{La}_{1-\mathrm{x}} \mathrm{Sr}_{\mathrm{x}} \mathrm{CoO}_{3-\delta}$ perovskite electrocatalysts. Nat. Commun. 7, 11053 (2016).

50. Raabe, S. et al. In situ electrochemical electron microscopy study of oxygen evolution activity of doped manganite perovskites. Adv. Funct. Mater. 22, 3378-3388 (2012).

51. Xu, X. et al. Co-doping strategy for developing perovskite oxides as highly efficient electrocatalysts for oxygen evolution reaction. Adv. Sci. 3, 1500187 (2016).

52. Zhu, Y. et al. Enhancing electrocatalytic activity of perovskite oxides by tuning cation deficiency for oxygen reduction and evolution reactions. Chem. Mater. 28, 1691-1697 (2016).

53. Petrie, J. R., Jeen, H., Barron, S. C., Meyer, T. L. \& Lee, H. N. Enhancing perovskite electrocatalysis through strain tuning of the oxygen deficiency. J. Am. Chem. Soc. 138, 7252-7255 (2016).

54. Chen, C.-F. et al. Oxygen-deficient $\mathrm{BaTiO}_{3-x}$ perovskite as an efficient bifunctional oxygen electrocatalyst. Nano Energy 13, 423-432 (2015).

55. Liu, Z.-Q., Cheng, H., Li, N., Ma, T. Y. \& Su, Y.-Z. $\mathrm{ZnCo}_{2} \mathrm{O}_{4}$ quantum dots anchored on nitrogen-doped carbon nanotubes as reversible oxygen reduction/evolution electrocatalysts. Adv. Mater. 28, 3777-3784 (2016).

56. Liu, X. et al. Hierarchical $\mathrm{Zn}_{\mathrm{x}} \mathrm{Co}_{3-\mathrm{x}} \mathrm{O}_{4}$ nanoarrays with high activity for electrocatalytic oxygen evolution. Chem. Mater. 26, 1889-1895 (2014).

57. Li, Y. et al. Nitrogen-doped graphene quantum dots with oxygen-rich functional groups. J. Am. Chem. Soc. 134, 15-18 (2012).

58. Liang, H. et al. Hydrothermal continuous flow synthesis and exfoliation of NiCo layered double hydroxide nanosheets for enhanced oxygen evolution catalysis. Nano Lett. 15, 1421-1427 (2015).
59. Li, B.-Q., Tang, C., Wang, H.-F., Zhu, X.-L. \& Zhang, Q. An aqueous preoxidation method for monolithic perovskite electrocatalysts with enhanced water oxidation performance. Sci. Adv. 2, e1600495 (2016).

60. Lee, D. U., Kim, B. J. \& Chen, Z. One-pot synthesis of a mesoporous $\mathrm{NiCo}_{2} \mathrm{O}_{4}$ nanoplatelet and graphene hybrid and its oxygen reduction and evolution activities as an efficient bi-functional electrocatalyst. J. Mater. Chem. A 1, 4754-4762 (2013).

61. Song, F., Schenk, K. \& Hu, X. A nanoporous oxygen evolution catalyst synthesized by selective electrochemical etching of perovskite hydroxide CoSn $(\mathrm{OH})_{6}$ nanocubes. Energy Environ. Sci. 9, 473-477 (2016).

62. Risch, M. et al. Structural changes of cobalt-based perovskites upon water oxidation investigated by EXAFS. J. Phys. Chem. C 117, 8628-8635 (2013).

63. Kanan, M. W. et al. Structure and valency of a cobalt-phosphate water oxidation catalyst determined by in situ X-ray spectroscopy. J. Am. Chem. Soc. 132, 13692-13701 (2010).

64. Grimaud, A. et al. Activating lattice oxygen redox reactions in metal oxides to catalyse oxygen evolution. Nat. Chem. 9, 457-465 (2017).

65. Grimaud, A., Hong, W. T., Shao-Horn, Y. \& Tarascon, J. M. Anionic redox processes for electrochemical devices. Nat. Mater. 15, 121-126 (2016).

\section{Acknowledgements}

This work was supported by National Key Research and Development Program (No. 2016YFA0202500 and 2016YFA0200102), Natural Scientific Foundation of China (No 21422604), and Tsinghua University Initiative Scientific Research Program. We thank Shu-Yuan Zhang, Chen-Yu Chen, Dr. Xiaoyang Cui, and Dr. Bin Wang for helpful discussion.

\section{Author contributions}

Q.Z. conceived the study and supervised the work. Q.Z. and B.-Q.L. designed the experiments. B.-Q.L. and Z.-J.X. synthesized the electrocatalysts and evaluated the water oxidation performance, B.Z. carried out TEM analysis, C.T. and H.-F.W. carried out structural and composition analysis of the electrocatalyst. B.-Q.L. and Q.Z. wrote the paper. All authors contributed to the discussion.

\section{Additional information}

Supplementary Information accompanies this paper at doi:10.1038/s41467-017-01053-X.

Competing interests: The authors declare no competing financial interests.

Reprints and permission information is available online at http://npg.nature.com/ reprintsandpermissions/

Publisher's note: Springer Nature remains neutral with regard to jurisdictional claims in published maps and institutional affiliations.

Open Access This article is licensed under a Creative Commons Attribution 4.0 International License, which permits use, sharing, adaptation, distribution and reproduction in any medium or format, as long as you give appropriate credit to the original author(s) and the source, provide a link to the Creative Commons license, and indicate if changes were made. The images or other third party material in this article are included in the article's Creative Commons license, unless indicated otherwise in a credit line to the material. If material is not included in the article's Creative Commons license and your intended use is not permitted by statutory regulation or exceeds the permitted use, you will need to obtain permission directly from the copyright holder. To view a copy of this license, visit http://creativecommons.org/ licenses/by/4.0/.

(C) The Author(s) 2017 